\section{Inguinal Ulceroglandular Tularemia Caused by Francisella tularensis Subspecies holarctica, Canada}

\author{
Carl Boodman, Quinlan Richert, Sylvain Lother, \\ Ken Kasper, Sergio Fanella, Philippe Lagacé-Wiens, \\ Yoav Keynan
}

\begin{abstract}
Author affiliations: University of Manitoba, Winnipeg, Manitoba, Canada; Shared Health Inc., Winnipeg (P. Lagacé-Wiens)
\end{abstract}

DOI: https://doi.org/10.3201/eid2704.203262

Tularemia is a zoonotic disease caused by the gramnegative coccobacillus Francisella tularensis, a Biosafety Level 3 pathogen and potential agent of bioterrorism. We describe 2 cases of perigenital ulcer disease caused by Francisella tularensis subspecies holarctica in Manitoba, Canada. These cases caused inadvertent exposure among laboratory personnel.

$I^{n}$ n July 2018, a previously healthy girl 4 years of age Iwas brought to the Health Sciences Centre at University of Manitoba (Winnipeg, Manitoba, Canada) for fever, right inguinal swelling, and dysuria. The patient's symptoms had worsened despite completing a 5-day course of trimethoprim/sulfamethoxazole prescribed by her family doctor for a presumed urinary tract infection 1 week before admission. The patient lived on a rural property bordered by forest in southern Manitoba, Canada. She enjoyed playing with her dogs and cats and often returned from the yard with ticks embedded in her skin.

At admission, her vital signs were within normal limits. We noted a small ulcer lateral to the right labia majora. This shallow nonpurulent ulcer was $<1 \mathrm{~cm}$ long, surrounded by erythema, and accompanied by tender local lymphadenopathy. We took a swab sample of the ulcer for bacterial culture and prescribed ceftriaxone for presumed cellulitis.

Three days after admission, the culture revealed pinpoint growth of Francisella tularensis on chocolate agar, identified by matrix-assisted laser desorption/ ionization-time of flight mass spectrometry (Bruker, https://www.bruker.com) (1). However, because a Biosafety Level 3 (BSL-3) pathogen had not been suspected, the culture was manipulated outside a biosafety cabinet (BSC). The exposed laboratory technologist was prescribed oral doxycycline $(100 \mathrm{mg} 2 \times / \mathrm{d}$ for 14 days) as postexposure prophylaxis; the technician showed no signs or symptoms of tularemia. The bacterial isolate was classified as a UN2814, category
A infectious substance; it was mailed in a sealed container with polystyrene foam-insulated packaging and an established Emergency Response Assistance Plan and placed in a box displaying the biohazard symbol to Canada's National Microbiology Laboratory (NML) for subspeciation. The NML identified the isolate as Francisella tularensis type B subspecies holarctica. We treated the patient for ulceroglandular tularemia $(20 \mathrm{mg} / \mathrm{kg}$ of oral ciprofloxacin $2 \times / \mathrm{d}$ for 14 days), prompting a complete recovery.

In August 2019, a woman 60 years of age arrived at Brandon Regional Health Centre (Brandon, Manitoba, Canada) with acute onset of hypotension and an ulcer beside her right labia majora. She had had chills for several days before seeking care. She had endstage renal disease managed by hemodialysis and sick sinus syndrome managed by a pacemaker. The patient lived in a rural area of southern Manitoba and had found a tick attached to her abdomen $\approx 1$ week before admission. She was not sure how long the tick had been attached; she removed it upon discovery.

At admission, the patient was hypotensive (70/25 $\mathrm{mm} \mathrm{Hg}$ ). She had a paced heart rate of $60 \mathrm{bpm}$ and oxygen saturation of $99 \%$ on room air. She did not have a fever. We noted a $2 \mathrm{~cm}$ long ulcer beside the right labia majora with surrounding erythema and bilateral inguinal lymphadenopathy. We found a $2 \mathrm{~cm}$ long necrotic eschar with surrounding erythema at the site of tick attachment.

The gram stain cultured from the perivulvar ulcer showed no organisms. However, faint growth appeared on chocolate agar on day 3. We identified F. tularensis using matrix-assisted laser desorption/ ionization-time of flight mass spectrometry (1). Before speciation, the culture had been manipulated outside a BSC, resulting in the exposure of 1 technologist; this technologist received doxycycline for 14 days as prophylaxis and had no signs or symptoms of infection. The NML identified the sample as F. tularensis subspecies holarctica. The patient was treated with gentamicin ( $2 \mathrm{mg} / \mathrm{kg} 1 \times / \mathrm{d}$ for 7 days) and oral ciprofloxacin (500 mg $1 \times / \mathrm{d}$ for 14 days) and symptoms resolved. We did not conduct serologic tests on samples from either patient. We notified the medical health officer of both cases.

The low infectious dose and easy dissemination of $F$. tularensis pose a substantial risk for laboratoryacquired infections when manipulated outside of a BSC $(2,3)$. The perigenital localization of tularemia in these cases produced an especially hazardous situation for laboratory exposure; in contrast to blood, lymph node, and bone marrow samples, genital lesions are not usually suspected to harbor BSL-3 
pathogens. A history of animal or arthropod exposure is a risk factor that can alert laboratory staff to the possibility of tularemia, enabling the application of appropriate precautions (4). Pinpoint colonies of gram-negative coccobacilli growing aerobically on chocolate agar 48 hours after plating might indicate the presence of $F$. tularensis and should prompt BSL3 precautions, as emphasized by the Centers for Disease Control and Prevention's Laboratory Response Network in affiliation with the American Society for Microbiology $(5,6)$. Of 42 cases of laboratory-acquired tularemia documented by Overholt et al. (7), 16 were unsuspected by microbiologists and occurred outside of a known exposure.

These 2 cases caused by F. tularensis subspecies holarctica support veterinary studies suggesting that this subspecies might be more common in the Canadian prairies than the more virulent $F$. tularensis subspecies tularensis identified elsewhere in North America $(8-10)$. The milder symptoms associated with $F$. tularensis subspecies holarctica might require a higher index of clinical suspicion, especially among patients with exposure to arthropods or wild mammals.

\section{About the Author}

Dr. Boodman is an infectious disease and medical microbiology resident doctor at the University of Manitoba, Winnipeg. His research interests include neglected infectious diseases and the interplay between infectious disease and socioeconomic disparities.

\section{References}

1. Rudrik JT, Soehnlen MK, Perry MJ, Sullivan MM, Reiter-Kintz W, Lee PA, et al. Safety and accuracy of matrixassisted laser desorption ionization-time of flight mass spectrometry for identification of highly pathogenic organisms. J Clin Microbiol. 2017;55:3513-29.

2. Shapiro DS, Schwartz DR. Exposure of laboratory workers to Francisella tularensis despite a bioterrorism procedure. J Clin Microbiol. 2002;40:2278-81. https:/ / doi.org/10.1128/ JCM.40.6.2278-2281.2002

3. Centers for Disease Control and Prevention. Managing potential laboratory exposures to Francisella tularensis. 2018 [cited 2020 Oct 04]. https://www.cdc.gov/tularemia/ laboratoryexposure/index.html

4. Singh K. Laboratory-acquired infections. Clin Infect Dis. 2009;49:142-7. https://doi.org/10.1086/599104

5. Miller JM, Astles R, Baszler T, Chapin K, Carey R, Garcia L, et al. Guidelines for safe work practices in human and animal medical diagnostic laboratories: recommendations of a CDCconvened, biosafety Blue Ribbon Panel. [Erratum in: MMWR Surveill Summ. 2012;61:214]. MMWR Suppl. 2012;61:1-102.

6. Craft D, Kijek T. Sentinel level clinical laboratory guidelines for suspected agents of bioterrorism and emerging infectious diseases: Francisella tularensis. 2016 [cited 2020 Oct 3]. https://asm.org/ASM/media/Policy-and-Advocacy/ LRN/Sentinel\%20Files/tularemia.pdf
7. Overholt EL, Tigertt WD, Kadull PJ, Ward MK, David CN, Rene RM, et al. An analysis of forty-two cases of laboratoryacquired tularemia: treatment with broad spectrum antibiotics. Am J Med. 1961;30:785-806. https:/ / doi.org/ 10.1016/0002-9343(61)90214-5

8. Wobeser G, Ngeleka M, Appleyard G, Bryden L, Mulvey MR. Tularemia in deer mice (Peromyscus maniculatus) during a population irruption in Saskatchewan, Canada. J Wildl Dis. 2007;43:23-31. https:// doi.org/10.7589/0090-3558-43.1.23

9. Wobeser G, Campbell GD, Dallaire A, McBurney S. Tularemia, plague, yersiniosis, and Tyzzer's disease in wild rodents and lagomorphs in Canada: a review. Can Vet J. 2009;50:1251-6.

10. Antonation KS, Bekal S, Côté G, Dallaire A, Corbett CR. Multiple-locus variable-number tandem-repeat analysis of Francisella tularensis from Quebec, Canada. Lett Appl Microbiol. 2015;60:328-33. https://doi.org/10.1111/lam.12371

Address for correspondence: Carl Boodman, Room 543, Basic Medical Sciences Building, 745 Bannatyne Avenue, Winnipeg, MB R3E 0J9, Canada; email: boodmanc@myumanitoba.ca

\section{Risk for Fomite-Mediated Transmission of SARS-CoV-2 in Child Daycares, Schools, Nursing Homes, and Offices}

\author{
Alicia N.M. Kraay, Michael A.L. Hayashi, \\ David M. Berendes, Julia S. Sobolik, Juan S. Leon, \\ Benjamin A. Lopman
}

Author affiliations: Emory University, Atlanta, Georgia, USA

(A.N.M. Kraay, J.S. Sobolik, J.S. Leon, B.A. Lopman); University of Michigan, Ann Arbor, Michigan, USA (M.A.L. Hayashi); Centers for Disease Control and Prevention, Atlanta (D.M. Berendes)

DOI: https://doi.org/10.3201/eid2704.203631

Severe acute respiratory syndrome coronavirus 2 can persist on surfaces, suggesting possible surface-mediated transmission of this pathogen. We found that fomites might be a substantial source of transmission risk, particularly in schools and child daycares. Combining surface cleaning and decontamination with mask wearing can help mitigate this risk. 